\title{
Prenatal maternal anxiety promotes atopic dermatitis in offspring via placental DNA methylation changes
}

\author{
Hyo-Bin Kim, ${ }^{1}$ Mi-Jin Kang, ${ }^{2}$ So-Yeon Lee, ${ }^{2}$ Yee-Jin Shin, ${ }^{3}$ Soo-Jong Hong ${ }^{2}$
}

\section{Abstract}

Background: Maternal anxiety during pregnancy has been previously reported to be associated with atopic dermatitis (AD) in offspring. The potential mechanism is not yet proven but epigenetic change may be suggested.

Objective: We examined whether maternal anxiety during pregnancy may alter placental DNA methylation, then develop $\mathrm{AD}$ in the offspring.

Methods: We evaluated maternal anxiety at 36 weeks of gestation by self-reported questionnaires, the State-Trait Anxiety Inventory-Trait subscale (STAI-T), in the Cohort for Childhood Origin of Asthma and Allergic Diseases (COCOA) study. AD was diagnosed at 6 months of age by pediatric allergists. We stratified the subjects into four groups according to the STAI score of mothers and diagnosis of $\mathrm{AD}$ in children. Placental genome-wide methylation microarray was analyzed using Infinium 450K BeadChip and selected genes were validated by pyrosequencing.

Results: From microarray, several differential methylation sites were identified in AD and healthy subjects and in total subjects, regarding to the STAI scores. Among differential methylation sites in microarray, six sites were selected for pyrosequencing. And site of matrix metalloproteinases 27 (MMP27) among 6 sites showed decreased methylation in AD infants with high STAI mothers compared to healthy infants with low STAI mothers.

Conclusion: Epigenetic change in placenta can be a suggesting mechanism for the development of AD in offspring at 6 months of age associated with maternal anxiety during pregnancy and MMP27 may be a candidate gene.

Key words: anxiety, atopic dermatitis, DNA methylation, epigenetic, pregnancy

\section{Citation:}

Kim, H. B., Kang, M. J., Lee, S. Y., Shin, Y. J., Hong, S. J. (2023)

Prenatal maternal anxiety promotes atopic dermatitis

in offspring via placental DNA methylation changes.

Asian Pac J Allergy Immunol, 41(1), 60-66.

https://doi.org/10.12932/ap-221119-0699

\footnotetext{
Affiliations:

Department of Pediatrics, Inje University Sanggye Paik Hospital, Seoul, Republic of Korea

2 Department of Pediatrics, Childhood Asthma Atopy Center, Environmental Health Center, Asan Medical Center, University of Ulsan College of Medicine, Seoul, Republic of Korea Department of Psychiatry and Behavioral Sciences, Severance Hospital, Yonsei University College of Medicine, Seoul, Republic of Korea
}

\author{
Corresponding author: \\ Soo-Jong Hong \\ Department of Pediatrics, Childhood Asthma Atopy Center, \\ Environmental Health Center, Asan Medical Center, \\ University of Ulsan College of Medicine, \\ 88 Olympic-ro-43-gil Songpa-gu, Seoul 05505, Republic of Korea \\ E-mail: sjhong@amc.seoul.kr
}

\section{Introduction}

Epidemiological studies of atopic dermatitis (AD) around the world have revealed geographical differences in its prevalence $^{1,2}$ and it is still having increasing tendency of this disorder in Korean children. ${ }^{3} \mathrm{AD}$ is a common allergic skin disease in childhood and represents the first step of an atopic march. Identifying the risk factors and the mechanisms underlying its development is therefore important for the prevention of $\mathrm{AD}$ itself and also for the consequent development of respiratory allergic diseases. Many recent studies have evaluated the risk factors for allergic diseases during prenatal period, regarded as a critical time point for immune modulation. ${ }^{4-6}$ 
It has been reported that prenatal maternal psychological distress could be a risk for the development of $\mathrm{AD}^{7,8}$ We have also previously reported that prenatal maternal distress is associated with $\mathrm{AD}$ in offspring, ${ }^{9}$ but the mechanisms of this had not yet been described. Oxidative stress is one of the suggested underlying mechanisms ${ }^{10}$ and additionally, epigenetic change could be an important mechanism. There were some epigenetic studies targeting $\mathrm{AD},{ }^{11}$ but the associated environmental factors causing epigenetic change is not revealed yet. We therefore examined in our current study whether maternal anxiety during pregnancy may alter placental DNA methylation, then that subsequently contribute to the pathogenesis of $\mathrm{AD}$ in offspring.

\section{Methods}

\section{Study population}

Subjects were selected from the Cohort for Childhood Origin of Asthma and Allergic Diseases (COCOA), a prospective birth cohort study designed to identify early risk factors for allergic diseases in children. ${ }^{12}$ This study was approved by the Institutional Review Boards of Asan Medical Center (IRB no. 2008-0616), Samsung Medical Center (IRB no. 2009-02-021), Severance Hospital (IRB no. 4-2008-0588), the CHA Medical Center (IRB no. 2010-010), and the Seoul National University Hospital (IRB no. H-1401 -086-550). Written informed consent was obtained from the parents of each infant. The COCOA study evaluated prenatal maternal anxiety at 36 weeks of gestation using the State-Trait Anxiety Inventory-Trait subscale (STAI-T), a 20-item self-reporting questionnaire. STAI-T is scored on a 4-point Likert-type scale that reflects a general tendency to be anxious. The score ranges from 20 to 80 , with a higher score indicating a more severe anxiety level. The Korean version of STAI has been previously shown to exhibit excellent psychometric properties. ${ }^{13}$ Subjects with scores in the highest $25^{\text {th }}$ percentile were defined as being anxious in the present study. ${ }^{9} \mathrm{AD}$ was diagnosed at 6 months of age in offspring by pediatric allergists using a detailed history and physical examination on the basis of the Hanifin and Rajka criteria. $^{9}$

\section{Placental DNA methylation microarray}

For placental genome-wide methylation microarray, we stratified mother-baby dyads in accordance with the maternal STAI-T scores and diagnosis of offspring's AD at 6 months of age, then selected 17 dyads which could represent each group; i.e. four from low STAI mothers with no offspring's AD group, six from low STAI mothers with offspring's AD group, four from high STAI mothers with no offspring's AD group and 3 from high STAI mothers with offspring's AD group (Table 1). Genomic DNA was isolated from the placental tissue using the Gentra Puregene Blood Kit (QIAGEN, Redwood City, CA, USA) in accordance with the manufacturer's protocol. Bisulfite conversion of the extracted DNA was conducted using the EZ DNA Methylation-Lightning kit (Zymo Research, Irvine, CA, USA). The converted samples were then assayed using Infinium Human Methylation 450 BeadChip kits (Illumina, San Diego, CA, USA). Image and data analysis of these chips were performed using an Illumina iScan scanner. Each methylation data point was represented by fluorescent signals from the $M$ (methylated) and $U$ (unmethylated) alleles. The background intensity was computed from a set of negative controls and was subtracted from each analytical data point. The fluorescent signal ratio for the two alleles was then computed as $B=(\max (M, 0)) /(|\mathrm{U}|+|\mathrm{M}|+100)$. The $B$-value reflects the methylation level of each $\mathrm{CpG}$ site. A $ß$-value range of $0-1$ represented 0 to $100 \%$ methylation, respectively, for each $\mathrm{CpG}$ site. Array data export processing and analysis was performed using Illumina GenomeStudio v2011.1 (Methylation Module v1.9.0) and R 3.0.2. CpG sites with a $P$-value $<0.05$ and a mean methylation change $>0.2$ were considered to be differentially methylated.

\section{Pyrosequencing}

Pyrosequencing of 100 additional placental tissue samples was performed to validate the microarray results. This sample population comprised the following: forty-one from low STAI mothers with no offspring's AD group, eleven from low STAI mothers with offspring's AD group, forty-three from high STAI mothers with no offspring's AD group and 5 from high STAI mothers with offspring's AD group (Table 2).

Table 1. Clinical characteristics of the subjects selected for genome-wide methylation microarray

\begin{tabular}{|c|c|c|c|c|c|}
\hline & STAI (low) \& AD (-) & STAI (low) \& AD (+) & STAI (high) \& AD (-) & STAI (high) \& AD (+) & $p$-value \\
\hline Number of subjects & 4 & 6 & 4 & 3 & \\
\hline Maternal age (years)* & $33.5 \pm 3.0$ & $31.8 \pm 3.1$ & $33.5 \pm 4.8$ & $34.7 \pm 3.8$ & 0.763 \\
\hline GA at birth (weeks)* & $38.6 \pm 0.4$ & $39.9 \pm 1.0$ & $38.1 \pm 0.8$ & $38.9 \pm 1.4$ & 0.116 \\
\hline Birth weight $(\mathrm{g})^{*}$ & $3096.3 \pm 487.0$ & $3299.2 \pm 377.2$ & $3132.5 \pm 401.7$ & $3465.0 \pm 388.7$ & 0.613 \\
\hline Child's sex (male:female) & $4: 0$ & $6: 0$ & $4: 0$ & $3: 0$ & NA \\
\hline Pre-pregnancy body mass index $\left(\mathrm{kg} / \mathrm{m}^{2}\right)^{*}$ & $19.7 \pm 1.4$ & $20.3 \pm 1.5$ & $22.4 \pm 1.6$ & $22.3 \pm 2.4$ & 0.031 \\
\hline Parental history of allergic diseases $(\%)^{\#}$ & 100.0 & 83.3 & 75.0 & 33.3 & 0.678 \\
\hline STAI score at GA 36 weeks* & $33.5 \pm 5.0$ & $31.5 \pm 7.3$ & $54.3 \pm 10.6$ & $50.0 \pm 12.2$ & 0.008 \\
\hline
\end{tabular}

${ }^{*}$ Values are shown as a mean \pm standard deviation; STAI, State-Trait Anxiety Inventory-Trait subscale; GA, Gestational age; AD, atopic dermatitis $P$-values were determined using the Kruskal-Wallis Test and ${ }^{*}$ chi-square test 
Table 2. Clinical characteristics of the subjects analyzed by pyrosequencing

\begin{tabular}{|c|c|c|c|c|c|}
\hline & STAI (low) \& AD (-) & STAI (low) \& AD (+) & STAI (high) \& AD (-) & STAI (high) \& AD (+) & $p$-value \\
\hline Number & 41 & 11 & 43 & 5 & \\
\hline Maternal age $(\text { years })^{*}$ & $32.8 \pm 4.3$ & $33.5 \pm 2.2$ & $32.6 \pm 4.1$ & $33.4 \pm 3.6$ & 0.769 \\
\hline GA at birth (weeks)* & $39.3 \pm 1.3$ & $39.4 \pm 0.7$ & $39.5 \pm 0.9$ & $39.4 \pm 1.7$ & 0.953 \\
\hline Birth weight $(\mathrm{g})^{*}$ & $3197.3 \pm 364.5$ & $3387.7 \pm 255.6$ & $3256.0 \pm 311.2$ & $3213.0 \pm 195.2$ & 0.333 \\
\hline Child's sex (male:female) & $21: 20$ & $8: 3$ & $21: 22$ & $2: 3$ & 0.500 \\
\hline Pre-pregnancy body mass index $\left(\mathrm{kg} / \mathrm{m}^{2}\right)^{*}$ & $20.5 \pm 2.8$ & $19.3 \pm 1.4$ & $21.1 \pm 2.2$ & $21.3 \pm 2.5$ & 0.081 \\
\hline Parental history of allergic diseases $(\%)^{\#}$ & 48.8 & 63.6 & 42.9 & 100.0 & 0.082 \\
\hline STAI score at GA 36 weeks ${ }^{*}$ & $29.0 \pm 4.4$ & $33.1 \pm 5.8$ & $50.2 \pm 6.8$ & $53.4 \pm 9.1$ & $<0.001$ \\
\hline
\end{tabular}

*Values are shown as a mean \pm standard deviation; STAI, State-Trait Anxiety Inventory-Trait subscale; GA, Gestational age; AD, atopic dermatitis

$P$-values were determined using the Kruskal-Wallis Test and "chi-square test

Briefly, genomic DNA was isolated from the placental tissue of these subjects as described above and bisulfite-converted. Aliquots of $200 \mathrm{ng}$ of these converted samples were then amplified using a PCR premix (Enzynomics, Seoul, Korea) in accordance with the manufacturer's protocol. The cycling conditions were as follows: 1 cycle at $95^{\circ} \mathrm{C}$ for 10 minutes for the initial denaturation step followed by 45 cycles of denaturation at $95^{\circ} \mathrm{C}$ for 30 seconds, various annealing temperatures $\left(58-68^{\circ} \mathrm{C}\right)$ for 30 seconds, and an extension at $72^{\circ} \mathrm{C}$ for 30 seconds. The reaction was terminated after a final cycle at $72^{\circ} \mathrm{C}$ for 5 minutes. Pyrosequencing was then performed using the PyroGold reagent kit (QIAGEN) and PyroMark ID machine (QIAGEN) in accordance with the manufacturer's instructions. The forward and reverse PCR primers and sequencing primers for specific CpGs are listed in Table S1.

\section{Network analysis}

Network analysis, using QIAGEN's Ingenuity Pathway Analysis system (IPA ${ }^{\oplus}$, QIAGEN, Redwood City, CA, USA, www.qiagen.com/ingenuity), was performed to gain further insight into the potential mechanisms underlying the effects of prenatal maternal stress on $\mathrm{AD}$ development in offspring. The highest-scoring network revealed a significant link with Cardiovascular Disease, Cell-To-Cell Signaling and Interaction and Cellular Growth and Proliferation.

\section{Statistical analysis}

Independent t-tests were used to examine differences in the DNA methylation levels between groups. All statistical analyses were performed with SPSS software (version 22: Chicago, IL, USA) and a $p<0.05$ was considered to indicate statistical significance.

\section{Results \\ Characteristics of the subjects}

Subjects in COCOA study were subgrouped depending on maternal STAI scores and presence of $\mathrm{AD}$ in offspring and seventeen subjects were selected for placental DNA methylation microarray. Maternal age, gestational age at birth, birth weight, sex of the offspring, and parental history of allergic disease were not statistically different among 4 groups (Table 1). Pre-pregnancy body mass index (BMI) was higher in high STAI group compared to low STAI group $(p=0.031)$.

In addition, one hundred subjects from 4 subgroups were selected for pyrosequencing and maternal age, gestational age at birth, birth weight, children's sex, parental history of allergic disease, and pre-pregnancy BMI were not statistically different among them (Table 2).

\section{Placental DNA methylation microarray}

Hierarchical cluster analysis of differentially methylated CpG sites between low and high STAI groups was performed in total, healthy and AD subjects (Figure S1). In total subjects, $29 \mathrm{CpG}$ sites were hypermethylated and 44 CpG sites were hypomethylated differentially between low and high STAI groups. In healthy subjects without $\mathrm{AD}$, there were 91 hypermethylated and 84 hypomethylated $\mathrm{CpG}$ sites differentially between low and high STAI groups, whereas in subjects with $\mathrm{AD}$, there were 147 hypermethylated and 191 hypomethylated CpG sites differentially between low and high STAI groups.

When intersecting placental DNA methylation change among groups, $A D A M T S 2$ and $R W D D 2 B$ were found to be differentially methylated between low and high STAI groups and C18orf1 was revealed differently between healthy and AD groups. Further, we analyzed for additional placental DNA methylation change which were differentially methylated between low and high STAI groups, and MAD1L1, MMP27, C18orf1, RWDD2B, MICAL3, and TSPAN7 genes have been found to be differentially methylated between low and high STAI. 
A
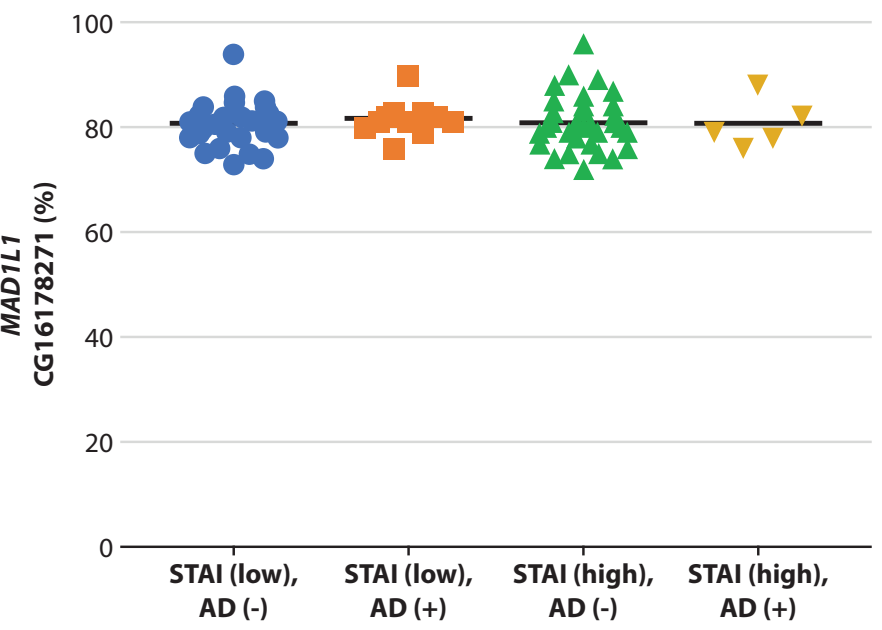

C

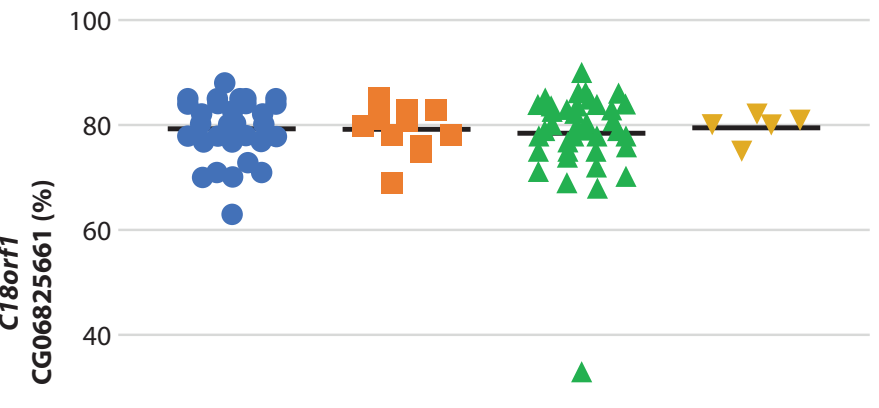

20

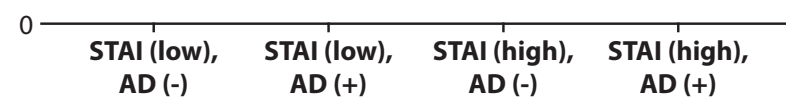

E

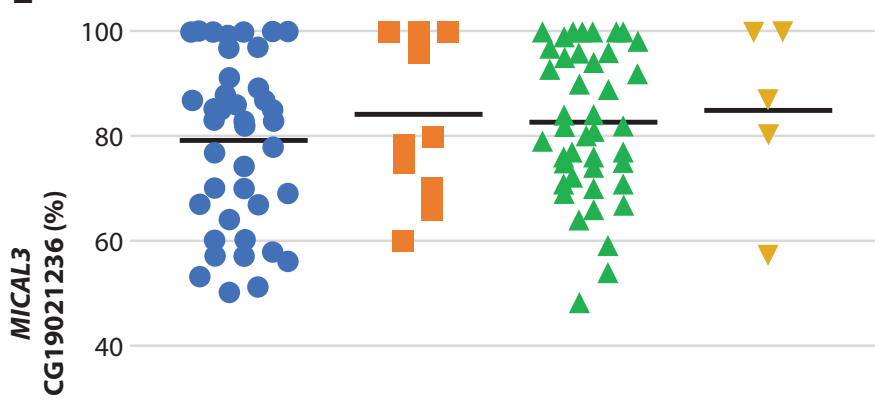

20

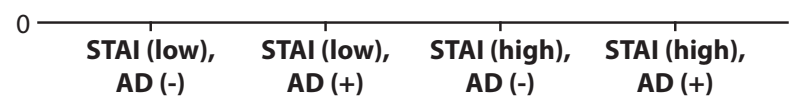

B

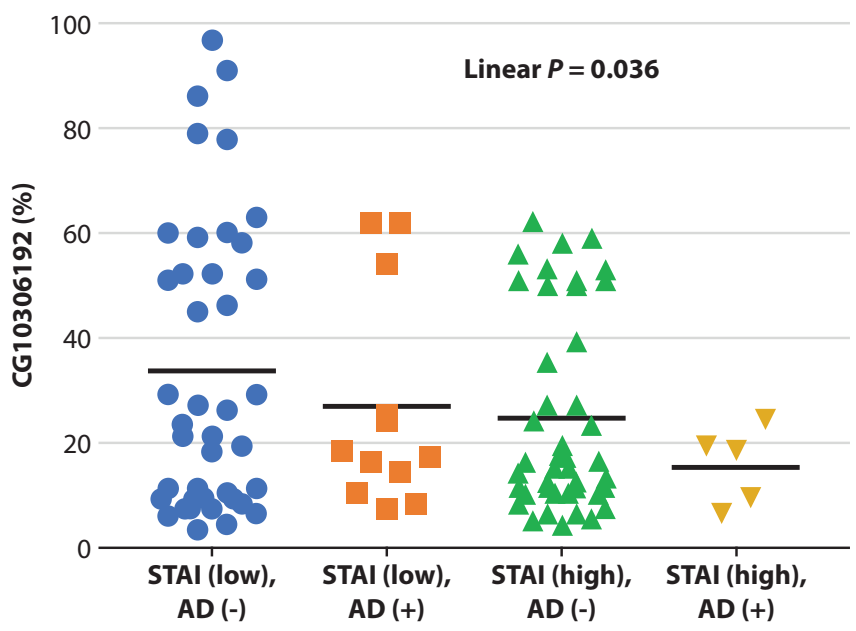

D

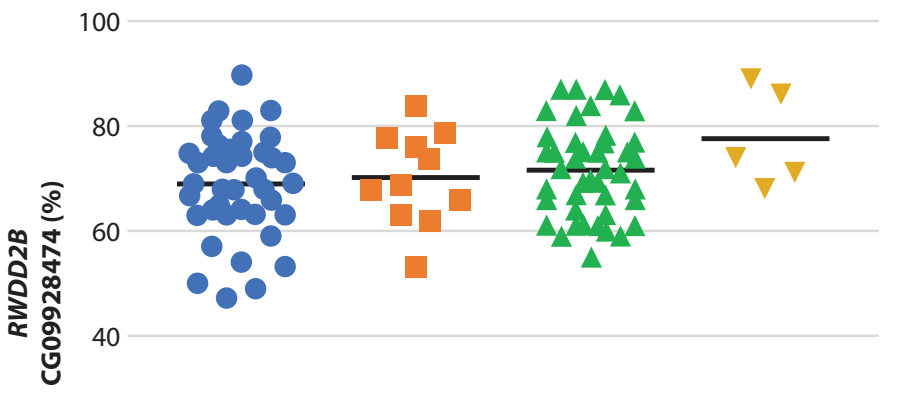

20

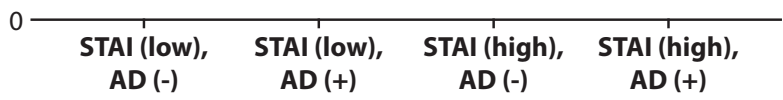

$\mathbf{F}$

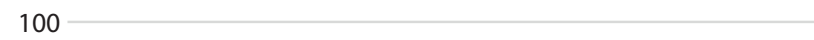

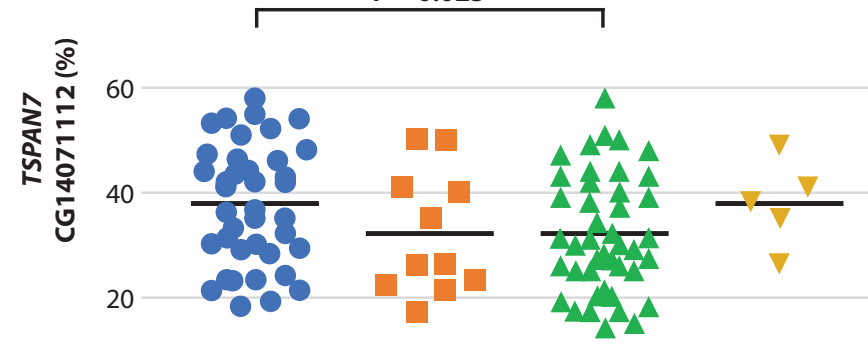

$\begin{array}{cccc}0 & \\ \text { STAI (low), } & \text { STAI (low), } & \text { STAI (high), } & \text { STAI (high), } \\ \text { AD (-) } & \text { AD (+) } & \text { AD (-) } & \text { AD (+) }\end{array}$

Figure 1. Pyrosequencing validation of six $\mathrm{CpG}$ sites selected from a genome-wide DNA methylation microarray. (A) MAD1L1. (B) MMP27. (C) C18orf1. (D) RWDD2B. (E) MICAL3. (F) TSPAN7. 

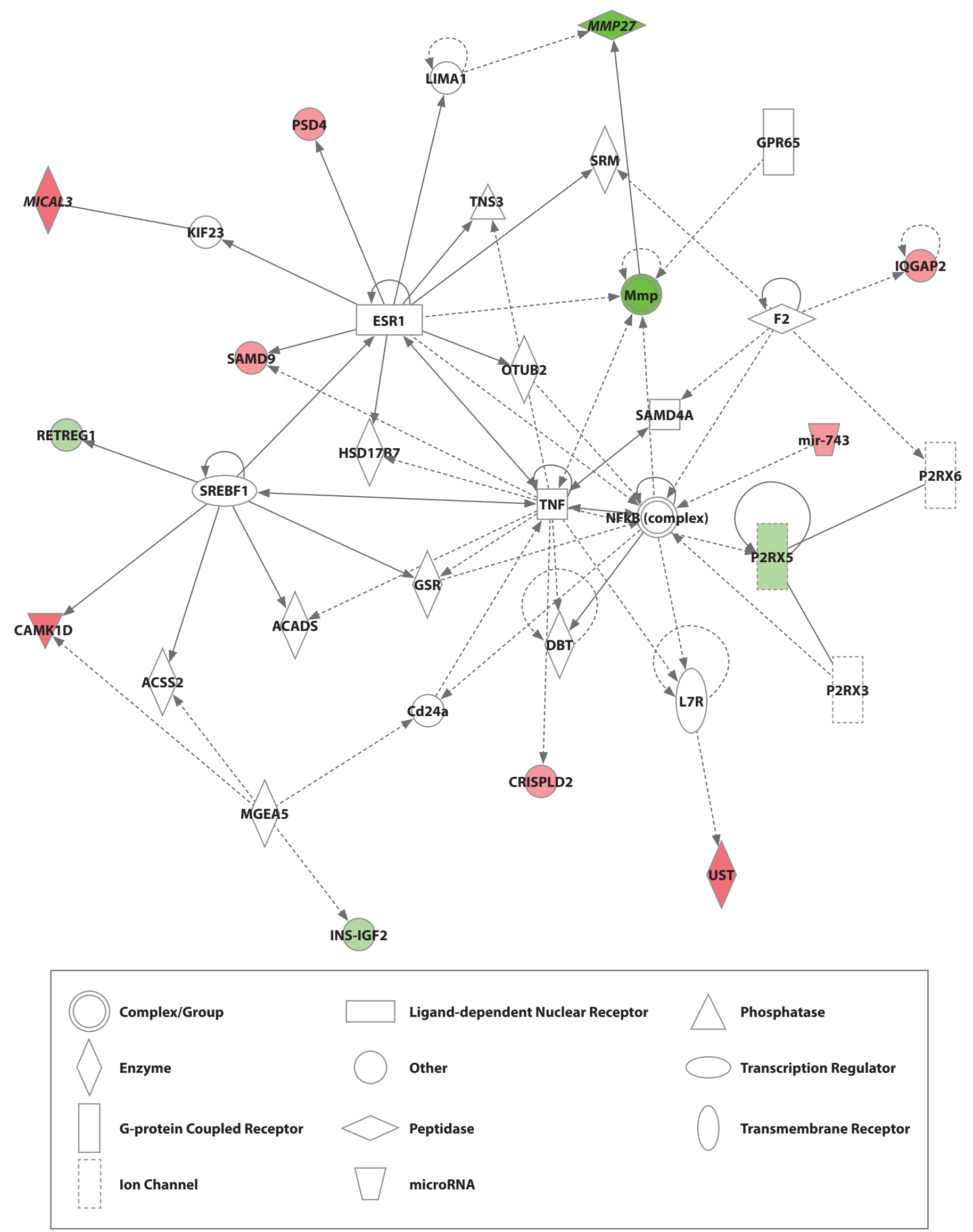

Figure 2. Molecular pathway analysis via the Ingenuity Pathways Analysis (IPA) system. Hypermethylated genes are indicated in red and hypomethylated genes in green. Nodes are denoted by various shapes that represent the functional classes of the proteins. Solid and dotted lines indicate direct and indirect interactions, respectively. 
Table 3. Genes selected for pyrosequencing validation

\begin{tabular}{|c|c|c|c|}
\hline Chromosome & Gene & CpG site & $\begin{array}{l}\text { Methylation status } \\
\text { (High STAI group compared to low STAI group) }\end{array}$ \\
\hline 7 & MAD1L1 & CG16178271 & Hyper \\
\hline 11 & MMP27 & CG10306192 & Нуро \\
\hline 18 & C18orf1 & CG06825661 & Hyper \\
\hline 21 & $R W D D 2 B$ & CG09928474 & Hyper \\
\hline 22 & MICAL3 & CG19021236 & Hyper \\
\hline $\mathrm{X}$ & TSPAN7 & CG14071112 & Нyро \\
\hline
\end{tabular}

\section{Pyrosequencing}

From the placental DNA methylation microarray, six genes were selected for the pyrosequencing (Table 3). DNA methylation of MMP27 was decreased in order as follows; from low STAI group without AD to low STAI group with $\mathrm{AD}$, high STAI group without $\mathrm{AD}$, and high STAI group with $\mathrm{AD}$ (Linear $p=0.036$ ) (Figure 1). Placental TSPAN7 methylation was higher in low STAI group without AD compared to high STAI group without AD $(p=0.025)$. Placental DNA methylation of MAD1L1, C18orf1, RWDD2B, and MICAL3 genes was not statistically different among 4 groups.

\section{Network analysis}

We additionally performed network analysis to gain a further insight into the potential mechanisms of the effects of prenatal maternal anxiety on offspring's AD. MMP27 showed a connection with TNF- $\alpha$ in this analysis (Figure 2).

\section{Discussion}

In this study, we investigated the epigenetic mechanism of the association between maternal anxiety during pregnancy and $\mathrm{AD}$ development in offspring at 6 months. Previously we reported that prenatal maternal distress is a risk factor for $\mathrm{AD}$ development in offspring and suggested that this is mediated by steroid imbalance and oxidative stress. We, herein, performed genome-wide DNA methylation microarray and pyrosequencing to prove epigenetic change in placenta for the association between maternal anxiety during pregnancy and $\mathrm{AD}$ development in the offspring. This response may be associated with DNA methylation of the MMP27 gene.

The MMPs are a group of zinc-dependent endopeptidases that degrade protein structures in the extracellular matrices of tissues. ${ }^{14}$ They play a key role in tissue remodeling and tissue infiltration by inflammatory cells. MMP27 is expressed and localized in the cycling human endometrium and reaches peak levels during the menstrual phase compared with the proliferative or secretory phases. The induction of MMP27 at the onset of menstruation has been shown to be responsible for tissue breakdown. MMP27 was also determined to be expressed by CD163+/ $\mathrm{CD} 206+$ macrophages in the human endometrium and in superficial endometrial lesions that exhibit considerable phenotypic plasticity in their various environments. ${ }^{15}$ Hence, pro- and anti-inflammatory states may depend on the environmental factors exposed to the placenta. We found that MMP27 CpG sites were hypomethylated in our study group with high maternal anxiety during pregnancy and showing $\mathrm{AD}$ onset in offspring compared to other groups. MMP27 expression in the placenta may therefore play a role in the development of $\mathrm{AD}$ in children as a result of a high maternal anxiety level during pregnancy.

According to the microarray result, we performed several network analyses, and MMP27 and MICAL3 showed the significant result. Furthermore $M M P 27$ was the gene that showed the significant methylation difference among the subgroups in the pyrosequencing. Therefore we suggested that MMP27 may play a role in the mechanism for the association between maternal anxiety and $\mathrm{AD}$ in the offspring, then focused on the MMP27. From the network analysis, MMP27 was suggested to be connected with TNF- $\alpha$ (Figure 2). Notably, TNF- $\alpha$ has previously been shown to have a crucial role in anxiety-like behavior and depression ${ }^{16}$ and to enhance MMP9 expression to promote shallow trophoblast invasion of the placenta. ${ }^{17}$ Taken together, the reports to date including our data suggest that an elevated TNF- $\alpha$ level in pregnant women with anxiety may induce higher MMP27 expression in the placenta, thereby promote inflammation and tissue remodeling leading to $\mathrm{AD}$ in offspring.

Our present study is the first report to link epigenetic changes to the association between prenatal maternal anxiety and $\mathrm{AD}$ development in young children. Previous reports in this area have mainly been epidemiological studies presenting an association between the maternal mental status and $\mathrm{AD}$ in children but with no mechanistic explanation. ${ }^{7,9}$ We now propose that differential placental DNA methylation of the MMP27 caused by prenatal maternal anxiety plays a role in this phenomenon. We also note that the aforementioned COCOA study was conducted by both allergy specialists and child psychologists from the beginning. Hence, validated psychological measures of maternal anxiety during pregnancy were used and $\mathrm{AD}$ in the children was properly diagnosed by the examination of pediatric allergy specialists to perform well-defined evaluation. 
There were some limitations in this study. We subgrouped our study subjects derived from the COCOA by prenatal maternal anxiety level and the presence of $\mathrm{AD}$ in children and then selected small numbers of these subjects for the analysis. This could have led to a selection bias. We tried however to ensure that we selected subjects who could be representative of each group. Further studies with larger numbers of subjects are needed.

In conclusion, the microenvironment that fetus had been exposed during pregnancy can be associated with $\mathrm{AD}$ development in offspring. Maternal anxiety during pregnancy may be an environmental risk factor for the development of $\mathrm{AD}$ and placental DNA methylation changes to the MMP27 may be a principal mechanism underlying this. Future studies are needed to elucidate the precise molecular mechanisms leading to the onset of allergic disease to develop preventative strategies.

\section{Conflicts of interest}

none

\section{Acknowledgements}

The authors sincerely thank Dr. Kangmo Ahn, Kyung Won Kim, Youn Ho Shin, Dong In Suh, Suk-Joo Choi, Ja-Young Kwon, Soo Hyun Kim, Jong Kwan Jun, Hye-Sung Won, Mi-Young Lee, Kyung-Sook Lee, Dankyu Yoon, and Jeom Kyu Lee for their contribution to the successful conduct of this study.

\section{Funding}

This work was supported by a Research Program funded by the Korea Centers for Disease Control and Prevention (2008-E33030-00, 2009-E33033-00， 2011-E33021-00, 2012 -E33012-00，2013-E51003-00，2014-E51004-00，2014-E51004 -01, 2014-E51004-02, 2017-E67002-00, 2017-E67002-01). The data analyzed in this study were obtained from the Chronic Disease Prevention \& Control Program (3334-308) which was funded by a grant from the Korea Centers for Disease Control and Prevention, Ministry of Health and Welfare, Republic of Korea.

\section{References}

1. Sybilski AJ, Raciborski F, Lipiec A, Tomaszewska A, Lusawa A, Samel-Kowalik P, et al. Atopic dermatitis is a serious health problem in Poland. Epidemiology studies based on the ECAP study. Postepy Dermatol Alergol. 2015;32:1-10.

2. Deckers IA, McLean S, Linssen S, Mommers M, van Schayck CP, Sheikh A. Investigating international time trends in the incidence and prevalence of atopic eczema 1990-2010: a systematic review of epidemiological studies. PLoS One. 2012;7:e39803.

3. Hong SJ, Ahn KM, Lee SY, Kim KE. The prevalences of asthma and allergic diseases in Korean children. Korean J Pediatr. 2008;51:343-50.

4. Burbank AJ, Sood AK, Kesic MJ, Peden DB, Hernandez ML. Environmental determinants of allergy and asthma in early life. J Allergy Clin Immunol. 2017;140:1-12.

5. Cai J, Zhao Y, Liu P, Xia B, Zhu Q, Wang X, et al. Exposure to particulate air pollution during early pregnancy is associated with placental DNA methylation. Sci Total Environ. 2017;607-608:1103-8.

6. Christensen S, Jaffar Z, Cole E, Porter V, Ferrini M, Postma B, et al Prenatal environmental tobacco smoke exposure increases allergic asthma risk with methylation changes in mice. Environ Mol Mutagen. 2017;58:423-33.

7. Hartwig IR, Sly PD, Schmidt LA, van Lieshout RJ, Bienenstock J, Holt PG, et al. Prenatal adverse life events increase the risk for atopic diseases in children, which is enhanced in the absence of a maternal atopic predisposition. J Allergy Clin Immunol. 2014;134:160-9.

8. Yoon J, Kim EM, Lee MY, Jung S, Cho HJ, Kim Y, et al. Perinatal maternal negative life events as risk factors of atopic dermatitis in female offspring. Ann Allergy Asthma Immunol. 2018;121:641-2.

9. Chang HY, Suh DI, Yang SI, Kang MJ, Lee SY, Lee E, et al. Prenatal maternal distress affects atopic dermatitis in offspring mediated by oxidative stress. J Allergy Clin Immunol. 2016;138:468-75.

10. Suh DI, Chang HY, Lee E, Yang SI, Hong SJ. Prenatal maternal distress and allergic diseases in offspring: review of evidence and possible pathways. Allergy Asthma Immunol Res. 2017;9:200-11.

11. Bin L, Leung DY. Genetic and epigenetic studies of atopic dermatitis. Allergy Asthma Clin Immunol. 2016;12:52.

12. Yang HJ, Lee SY, Suh DI, Shin YH, Kim BJ, Seo JH, et al. The Cohort for Childhood Origin of Asthma and allergic diseases (COCOA) study: design, rationale and methods. BMC Pulm Med. 2014;14:109.

13. Hahn D, Lee C, Chon K. Korean adaptation of Spielberger's STAI (K-STAI). Korean J Heal Psychol. 1996;1:1-14.

14. Gueders MM, Foidart JM, Noel A, Cataldo DD. Matrix metalloproteinases (MMPs) and tissue inhibitors of MMPs in the respiratory tract: potential implications in asthma and other lung diseases. Eur J Pharmacol. 2006;533:133-44.

15. Cominelli A, Gaide Chevronnay HP, Lemoine P, Courtoy PJ, Marbaix E, Henriet P. Matrix metalloproteinase-27 is expressed in CD163+/CD206+ M2 macrophages in the cycling human endometrium and in superficial endometriotic lesions. Mol Hum Reprod. 2014;20:767-75.

16. Karson A, Demirtaş T, Bayramgürler D, Balci F, Utkan T. Chronic administration of infliximab (TNF- $\alpha$ inhibitor) decreases depression and anxiety-like behavior in rat model of chronic mild stress. Basic Clin Pharmacol Toxicol. 2013;112:335-40.

17. Lockwood CJ, Oner C, Uz YH, Kayisli UA, Huang SJ, Buchwalder LF, et al. Matrix metalloproteinase 9 (MMP9) expression in preeclamptic decidua and MMP9 induction by tumor necrosis factor alpha and interleukin 1 beta in human first trimester decidual cells. Biol Reprod. 2008;78:1064-72. 